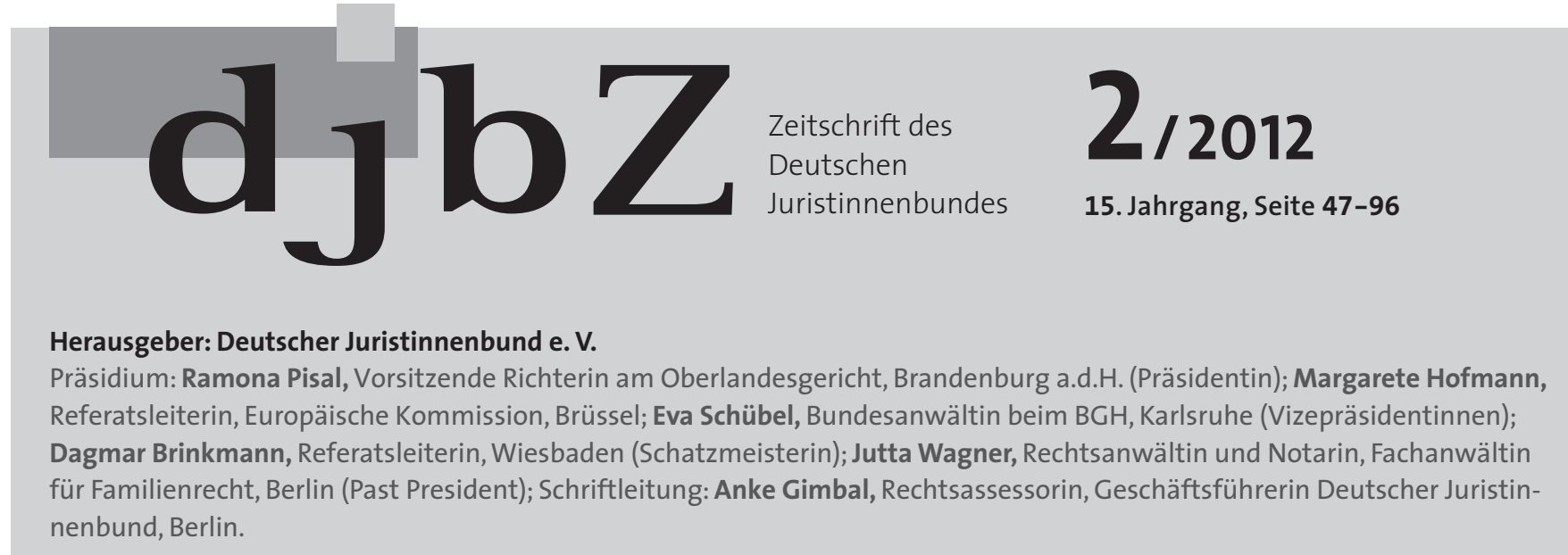

\title{
Aktionärinnen fordern Gleichberechtigung: Wie soll der Wandel gelingen?
}

\author{
19. März 2012, Königlich Norwegische Botschaft, Berlin
}

Den offiziellen Auftakt unseres 2012-Projektes „Aktionärinnen fordern Gleichberechtigung “, in dessen Rahmen wieder 75 Hauptversammlungen besucht werden, bildete unsere Veranstaltung „Wie soll der Wandel gelingen?“ Mit dieser Frage bat der djb am 19. März 2012 Expertinnen und Experten aus Politik und Wirtschaft in die Königlich Norwegische Botschaft in Berlin. Zunächst berichtete s. E. Botschaf- ter Sven Erik Svedman in seinem Grußwort von der norwegischen Erfahrung mit der Einführung der „Frauenquote“, danach sollten Empfehlungen zur Erreichung unseres Zieles, mehr Frauen in Führungspositionen börsennotierter Unternehmen zu bringen, ausgesprochen werden. Was dabei herauskam? Sechs starke Statements. (AH)

\section{No way but Norway's: nur die Quote hilft}

\section{Ramona Pisal \\ Präsidentin, Potsdam}

Sehr geehrter Herr Botschafter, sehr geehrte Damen und Herren Abgeordnete des Bundestages und der Landtage,

sehr geehrte Damen und Herren,

liebe Kolleginnen im djb,

es freut mich sehr, Sie zu unserer heutigen Veranstaltung in der Königlich Norwegischen Botschaft begrüßen zu dürfen.

Mein besonderer Dank gilt dabei Ihnen, Exzellenz, für Ihre Gastfreundschaft und die großzügige Überlassung des Veranstaltungsraumes hier in Ihrem Hause.

Bedanken möchte ich mich auch bei den Referentinnen und Referenten, die unserer Bitte, ein Statement abzugeben, gefolgt sind, sowie dem Bundesministerium für Familie, Senioren, Frauen und Jugend für die Förderung dieser Veranstaltung im Rahmen des Projektes „Aktionärinnen fordern Gleichberechtigung“.
Wir haben Sie heute eingeladen, weil wir gern von Expertinnen und Experten wissen möchten, wie wir unser Ziel erreichen können, mehr Frauen in die Aufsichtsräte, die Vorstände und generell in Führungspositionen der Unternehmen zu bringen.

Der wenig inspirierende Status quo zu diesem Thema ist allen Anwesenden hier hinlänglich bekannt. Sie alle wissen, dass Frauen in den 200 größten Unternehmen Deutschlands in Führungspositionen eklatant unterrepräsentiert sind. Auch 2011 fanden sich hier unverändert nur drei Prozent Frauen in den Vorständen und mit einer marginalen Steigerung nur knapp 12 Prozent in den Aufsichtsräten; mehr als zwei Drittel waren dabei von Arbeitnehmerseite entsandt. Dabei will ich es bewenden lassen. Die Einzelheiten finden sich ausführlich im Wochenbericht des DIW, 3/12, unter der Überschrift „Spitzengremien großer Unternehmen: Hartnäckigkeit männlicher Strukturen lässt kaum Platz für Frauen”. Darum die Frage an unsere Expertinnen und Experten:

„Wie soll der Wandel gelingen?” 
Schon 2010 und 2011 haben wir versucht unter Beteiligung der Wirtschaft darauf Antworten zu finden, indem wir jeweils 75 große börsennotierte Unternehmen besucht und als „Aktionärinnen" Fragen gestellt haben. Wir wollten gerne wissen, welche Ziele die Unternehmen für eine angemessene Repräsentanz von Frauen in Führungspositionen ansteuern und mit welchen strategischen Maßnahmen sie diese Ziele erreichen wollten. Die Ergebnisse dieser Befragungen waren mit Blick darauf wenig zufriedenstellend. Einzelheiten dazu finden Sie in zwei Veröffentlichungen dokumentiert. Die Studie 2010, die im Foyer ausliegt, enthält darüber hinaus eine Reihe von Beiträgen mit Vorschlägen zu der uns auch heute beschäftigenden Frage: Transparenz durch Berichtspflichten im Lagebericht zu dem Anteil der Frauen an den Beschäftigten, Zielvereinbarungen und Erfolgsvergütungen für Vorstandsmitglieder sowie Strategien und Konzepte zur Veränderung der Unternehmenskultur könnten jedenfalls flankierend geeignete Mittel sein?

Für unsere zweite Studie aus dem Jahr 2011, die hier auf Ihren Plätzen liegt, haben wir außer der Dokumentation und Auswertung der Besuche (von Hauptversammlungen, Anm. d.Red.) unsere europäischen Nachbarn befragt, wie sie das Problem gelöst haben oder lösen wollen. Über die Situation in Norwegen haben wir gleich drei Beiträge veröffentlichen können, denn Norwegen hat als erstes Land im Jahr 2003 ein Gesetz verabschiedet, das nach einer Übergangszeit die Unternehmen verbindlich verpflichtete, einen Frauenanteil in den Boards von 40 Prozent zu gewährleisten. Von Norwegen und aus seiner inzwischen langjährigen Erfahrung können wir darum besonders viel lernen, zum Beispiel:

- dass es ohne gesetzliche Verpflichtung nicht geht, denn die Unternehmen hatten die letzte Frist zur eigenen Abhilfe ungenutzt verstreichen lassen, ganz so wie unsere Wirtschaft seit der leeren Selbstverpflichtung im Jahr 2010;

- dass die Quote per Gesetz das Problem gezielt löst, entgegen reflexartig vorgebrachten anderslautenden Behauptungen, denn der gewünschte Frauenanteil von 40 Prozent ist dort erreicht;

v dass es genügend geeignete Frauen für diese Positionen gibt, denn binnen kurzem waren diese Frauen gefunden; das sollte auch in Deutschland gelingen;

- dass das Hauptproblem für Frauen in verantwortlicher Position wohl nicht die mangelnde Vereinbarkeit von Familie, gemeint sind wohl Kinder, und Beruf ist; denn diese Frauen sind ja nicht gegen ihren Willen und ihre Möglichkeiten zu diesen Aufgaben verpflichtet worden und bekommen ihren Alltag organisiert;

- dass „Quotenfrauen“ qualifiziert sind, denn ein Einbruch der norwegischen Wirtschaft ist seitdem nicht zu verzeichnen

- und vor allem die leicht bittere Erkenntnis, dass wohl kaum eine dieser offensichtlich qualifizierten Frauen jemals eine vergleichbare Position ohne die gesetzlich verordnete Quote hätte erreichen können.

Wie passt das in das Bild eines demokratisch verfassten Rechtsstaats, der von Verfassungs wegen Frauen die gleichen Rechte garantiert und sich zudem verpflichtet hat die Durch- setzung der Gleichberechtigung aktiv zu fördern? Wenn Sie mich fragen würden: gar nicht. Derselben Meinung war die seinerzeit konservative norwegische Regierung.

Unsere Erfahrungen in Deutschland nach all den Jahren, insbesondere seitdem wir uns mit dem Thema über das Projekt verstärkt beschäftigen, haben uns in der Überzeugung bestärkt, dass verlässliche Basis für einen Wandel nur ein Gesetz sein kann. Die Unternehmen haben bei den sogenannten Quotengipfeln keine Ziele für Aufsichtsräte und Vorstände genannt. Deutschland ist wie kaum ein anderes europäisches Land im Erwerbsleben von Rollenstereotypen geprägt. Das hat vor allem eine SinusStudie eindrucksvoll belegt. Einkommensstarke Positionen werden vielfach sicher oft unbewusst Männern vorbehalten, da mögen in Frage kommende Frauen noch so qualifiziert sein. Sie werden nach wie vor nur als Zuverdienerinnen angesehen. Vor diesem Hintergrund auf einen Mentalitätswechsel und einen Bewusstseinswandel zu setzen, erfordert mehr als die Geduld eines Gärtners. Darüber würden weitere vier bis fünf Jahrzehnte ins Land gehen, wie uns Frau Dr. Bankier bei unserer letzten Veranstaltung im November im Europäischen Haus vorgerechnet hat. Danach würde eine Quote von 40 Prozent im Vorstand bei den derzeitigen Zuwachsraten erst in 40 Jahren erreicht werden. Solange können und wollen wir einfach nicht mehr warten.

Heute stehen allerdings nicht unsere Untersuchungen und die Schlüsse daraus im Vordergrund, sondern die Meinung der von uns eingeladenen Expertinnen und Experten ist gefragt. Wir haben für Sie Kurzvitae bereit gelegt, so dass ich auf eine umfassende Vorstellung verzichten kann.

Wir sind sehr gespannt, welche Empfehlungen hier ausgesprochen werden.

Ich freue mich zunächst auf ein Grußwort von Ihnen, sehr geehrter Herr Botschafter Svedman. Dass Norwegen für uns ein Land mit Vorbildfunktion ist, habe ich schon zum Ausdruck gebracht. Dass es auch als einziges europäisches Land ein genderparitätisch besetztes Parlament hat, will ich aber auch nicht versäumen zu erwähnen.

Wir freuen uns auf Ihr Statement, sehr geehrter Herr Sattelberger: Ihr Unternehmen, die Deutsche Telekom, nimmt in Deutschland eine Vorbildfunktion ein und Sie werden sicher auch über Ihre Erfolge berichten. Da Sie uns schon bald wieder wegen anderer dringender Termine verlassen müssen, werden wir uns nach Ihrem Statement von Ihnen verabschieden.

Gespannt sind wir auf Ihre Empfehlung, die Sie, sehr geehrte Frau Dr. Lammers, uns aus der Sicht einer Partnerin der Zehnder International für den Personalbereich geben können.

Über Kurse für potentielle Aufsichtsrätinnen und die Datenbank qualifizierter Frauen beim Verband Deutscher Unternehmerinnen werden wir etwas von Ihnen, sehr geehrte Frau Bschorr, hören.

Daran wird sich anschließen ein Statement von Ihnen, sehr geehrte Frau Mast. Sie haben an einem Kurs des VdU für Aufsichtsrätinnen bereits teilgenommen.

Wir sind gespannt, welchen Rat Sie, sehr geehrte Frau Professorin Maier, uns als Direktorin des Harriett-Mill-Instituts geben können. 
Last but not least, liebe Monika, aus der Sicht von FidAR wird uns Frau Schulz-Strelow Ihre Empfehlungen vorstellen.

Wir freuen uns jetzt, Ihre Expertinnen- und Expertenmeinung zum Thema: „Wie soll der Wandel gelingen“ zu hören. Im Anschluss daran und nach einigen Worten des Dankes und zum Abschluss laden wir Sie herzlich zu einem Gedankenaustausch bei einem kleinen Imbiss ein.

Ich danke Ihnen für Ihre Aufmerksamkeit und freue mich jetzt zunächst auf Ihr Grußwort, Exzellenz.

\section{Grußwort}

\section{S.E. Botschafter Sven Erik Svedman \\ Berlin}

Sehr geehrte Frau Präsidentin Pisal, meine Damen und Herren,

ich freue mich, Sie heute zu dieser Veranstaltung in der norwegischen Botschaft und dem Felleshus der Nordischen Botschaften in Berlin begrüßen zu dürfen. Es ist mir eine besonders große Freude, dass so viele von Ihnen den Weg hierher gefunden haben, um an diesem Gedankenaustausch teilzunehmen.

Es sollte eigentlich keine Überraschung sein, dass es hier in Deutschland ein Interesse an den norwegischen und nordischen Ideen und gesellschaftlichen Entwicklungen gibt. In allen Bereichen sind die Beziehungen zwischen Deutschland und Norwegen eng, sowohl in der Politik, der Wirtschaft wie auch auf dem Gebiet der Kultur. Deutschland ist der wichtigste Partner Norwegens in Europa.

Trotz jahrelangen Kampfes um die Verbesserung der Gleichberechtigung zwischen Frauen und Männern sowohl in der Politik wie auch in der Wirtschaft ist das Thema heutzutage aktueller denn je, in Deutschland wie in Norwegen. Es gab in den letzten Jahrzehnten viele Fortschritte. Wir sollten aber auch erkennen, dass trotz der Fortschritte noch viele Herausforderungen bleiben.

Die Norwegerinnen und Norweger verstehen den Hintergrund und das Interesse für die Debatte über Gleichberechtigung zwischen Frauen und Männern hier in Deutschland sehr gut. Zum Beispiel diskutiert man hier heutzutage, ob man eine gesetzlich regulierte Quote in den Aufsichtsräten einführen soll, etwas was Norwegen in 2003 als erstes Land der Welt getan hat.

Wir sehen in der Debatte hier in Deutschland viele Gemeinsamkeiten mit der Debatte in Norwegen vor der Einführung der neuen Regelung in 2003. Die meisten, ob Sie in der Wirtschaft oder in der Politik tätig sind, sind sich in dem Ziel einig: Es ist wichtig, mehr Frauen in Führungspositionen und Aufsichtsräten zu haben. Die Meinungsverschiedenheiten bestehen aber darüber, wie das Ziel erreicht werden kann.

Dabei geht es aber um mehr als nur den Anteil von Frauen in Aufsichtsräten. Gerechtigkeit und Gleichstellung sind Grundwerte unserer Gesellschaft. Es gibt einen klaren positiven Zusammenhang zwischen der Berufstätigkeit der Frauen und der Entwicklung des Wachstums und des Wohlstandes in unserer Gesellschaft. Mit anderen Worten: Alle tüchtigen Menschen, unabhängig vom Geschlecht, müssen die gleichen Möglichkeiten erhalten, um Positionen zu bekommen, entweder im öffentlichen Dienst oder in der Wirtschaft.

Erlauben Sie mir in diesem Sinn einige Erfahrungen aus Norwegen einzubringen:

In Norwegen kündigte der damalige Wirtschaftsminister Ansgar Gabrielsen (Konservative Partei) im März 2002 an, dass sich die norwegische Regierung für eine gesetzlich festgelegte Forderung über die Repräsentation der Geschlechter in Aufsichtsräten einsetzen wird. Im April 2003, zwei Monate bevor der Antrag zur Verbesserung der Geschlechterbalance in Aufsichtsräten dem norwegischen Storting vorgelegt wurde, waren nur rund sieben Prozent der ständigen Aufsichtsratsmitglieder der damals 590 allgemeinen Aktiengesellschaften in Norwegen Frauen.

Die Forderung nach Geschlechterrepräsentation gilt in Norwegen heute für alle staatlichen Betriebe, das heißt für Gesellschaften, die vollständig in Staatsbesitz sind und für alle in Privatbesitz befindlichen allgemeinen Aktiengesellschaften. Die Vorschriften über die Repräsentation der Geschlechter sind in das allgemeine Aktiengesetz aufgenommen worden. Die Forderung verändert sich mit der Größe der Gesellschaft. Wenn die Gesellschaft aber mindestens zehn Mitglieder hat, soll jedes Geschlecht mit mindestens 40 Prozent repräsentiert sein. Im Dezember 2003 wurde das Gesetz durch eine breite Mehrheit im norwegischen Storting angenommen. Es wurde eine Ausnahme für private Aktiengesellschaften gemacht, weil viele private Aktiengesellschaften in Norwegen kleine Familienunternehmen sind, in denen die Eigentümer natürliche Personen sind, die selbst im Aufsichtsrat sitzen.

Was sind eigentlich die Ergebnisse in Norwegen ungefähr neun Jahre nach der Einführung der Frauenquote?

Zum einen: Der Frauenanteil in den Aufsichtsräten der allgemeinen Aktiengesellschaften ist seit dem damaligen Anteil von sieben Prozent bis auf 40 Prozent gestiegen. Mit anderen Worten haben die allgemeinen Aktiengesellschaften ihre Anforderungen erfüllt. Der Anteil von Frauen ist aber nicht höher als gesetzlich vorgeschrieben.

Auch der Anteil von Frauen als Aufsichtsratsvorsitzende in den allgemeinen Aktiengesellschaften ist gestiegen, aber nicht so wie der Anteil an weiblichen Aufsichtsratsmitgliedern. Der Anteil an weiblichen Vorsitzenden betrug in 2010 sieben Prozent, gegenüber nur drei Prozent in 2004. Mit an- 